\title{
Extensions of Büchi's problem: Questions of decidability for addition and $k$ th powers
}

\author{
by \\ Thanases Pheidas (Heraklion) and Xavier Vidaux (Concepción)
}

\begin{abstract}
We generalize a question of Büchi: Let $R$ be an integral domain, $C$ a subring and $k \geq 2$ an integer. Is there an algorithm to decide the solvability in $R$ of any given system of polynomial equations, each of which is linear in the $k$ th powers of the unknowns, with coefficients in $C$ ?

We state a number-theoretical problem, depending on $k$, a positive answer to which would imply a negative answer to the question for $R=C=\mathbb{Z}$.

We reduce a negative answer for $k=2$ and for $R=F(t)$, the field of rational functions over a field of zero characteristic, to the undecidability of the ring theory of $F(t)$.

We address a similar question where we allow, along with the equations, also conditions of the form " $x$ is a constant" and " $x$ takes the value 0 at $t=0$ ", for $k=3$ and for function fields $R=F(t)$ of zero characteristic, with $C=\mathbb{Z}[t]$. We prove that a negative answer to this question would follow from a negative answer for a ring between $\mathbb{Z}$ and the extension of $\mathbb{Z}$ by a primitive cube root of 1 .
\end{abstract}

1. Introduction. Given any $k=2,3, \ldots$, Büchi's question for $k$ (for short $\mathbf{B q}(k))$ is the following:

Question $1.1(\mathbf{B q}(k))$. Does there exist an algorithm to determine, given $m, n \in \mathbb{N}, A=\left(a_{i, j}\right)_{i, j} \in \mathcal{M}_{m, n}(\mathbb{Z})$ and $B=\left(b_{i}\right) \in \mathcal{M}_{m, 1}(\mathbb{Z})$, whether there exist $x_{1}, \ldots, x_{n} \in \mathbb{Z}$ satisfying the equations

$$
\sum_{j=1}^{n} a_{i, j} x_{j}^{k}=b_{i}, \quad i=1, \ldots, m,
$$

where, for any $r \in \mathbb{N}, \mathcal{M}_{m, r}(\mathbb{Z})$ is the set of $m \times r$ matrices with entries in $\mathbb{Z}$ ?

J. Richard Büchi asked the question for $k=2$ and this was made public by L. Lipshitz in [7]. The problem was investigated by Joseph Lipman and

2000 Mathematics Subject Classification: Primary 03C60; Secondary 12L05.

The second author acknowledges the hospitality of the University of Crete-Heraklion, where the main part of this work was done, while it was finished at Oxford University under the European "Marie Curie individual fellowship" MCFI-2002-00722. 
Barry Mazur (cf. [9]). Paul Vojta in [17] proved that a conjecture of Serge Lang implies a negative answer (we discuss this in Section 2).

It is obvious that, for any $k$, a negative answer to $\mathbf{B q}(k)$ would be a strong form of a negative answer to Hilbert's Tenth Problem (cf. [8] and [1]). In this paper:

- We show that for each odd $k \geq 3$ a negative answer to $\mathbf{B q}(k)$ would follow from a positive answer to a number-theoretical problem (Problem 2.2); the similar problem for $k=2$ was asked by Büchi. This is Theorem 2.4.

- We generalize Question $\mathbf{B q}(k)$ to any commutative ring $R$ and for any subring $C$ of allowed coefficients of equations (in this section).

- We show, using results of Vojta, that for $k=2$ the generalized problem for $R=F(t)$, the field of rational functions in the variable $t$ with coefficients in a field $F$ of zero characteristic, and for $C=\mathbb{Z}[t]$, has a negative answer if the existential ring theory of $F(t)$ in the language of rings augmented by $t$ is undecidable. These results are stated in Theorem 1.4 and Corollary 1.5 and their proofs are given in Section 3.

- We show that a question similar to 1.1 for $k=3$, for fields of rational functions of zero characteristic, will have a negative answer if $\mathbf{B q}(3)$ has a negative answer. The results are stated in Theorem 1.6 and Corollary 1.7 and proved in Sections 4 and 5 .

We generalize $\mathbf{B q}(k)$ to arbitrary integral domains as follows: Assume that $R$ is a commutative ring with a multiplicative identity, $C$ is a finitely generated subring of $R, k \in \mathbb{Z}$ and $k \geq 2$.

Question $1.2(\mathbf{B q}(k, R, C))$. Does there exist an algorithm to determine, given $m, n \in \mathbb{N}, A=\left(a_{i, j}\right)_{i, j} \in \mathcal{M}_{m, n}(C), B=\left(b_{i}\right) \in \mathcal{M}_{m, 1}(C)$ and a subset $J \subset\{1, \ldots, n\}$, whether there exist $x_{1}, \ldots, x_{n} \in R$ satisfying the equations

$$
\sum_{j=1}^{n} a_{i, j} x_{j}=b_{i}, \quad i=1, \ldots, m,
$$

and subject to the conditions: for $j \in J, x_{j} \in\left\{y^{k}: y \in R\right\}$ (for any $r \in \mathbb{N}$, $\mathcal{M}_{m, r}(C)$ is the set of $m \times r$ matrices with entries in $\left.C\right)$ ?

If $R=\mathbb{Z}$, it is trivial to see, using linear elimination, that $\mathbf{B q}(k, \mathbb{Z}, \mathbb{Z})$ is equivalent to $\mathbf{B q}(k)$.

We state Question 1.2 in the terminology of logic. For each $k \in \mathbb{N}$ we let $L_{k, C}$ denote the language which consists of the following symbols: (a) symbols for the elements of the ring $C$, (b) the symbol + for addition, (c) the predicate-symbol $P_{k}$ for the relation " $x$ is a $k$ th power", so $P_{k}(x) \leftrightarrow \exists y \in R$ $\left[x=y^{k}\right]$, (d) for each $c \in C$, a symbol for the function of multiplication by $c: x \mapsto c x$. We adopt the convention that we will always interpret these 
symbols in the stated way. Obviously, a positive-quantifier-free formula of $L_{k, C}$ is a disjunction of systems of linear equations of the type occurring in Question 1.2, together with conditions of the form $P_{k}\left(x_{i}\right)$. A positiveexistential formula of $L_{k, C}$ is a formula of the form $\exists y \phi(x, y)$ where $\phi$ is a positive-quantifier-free formula of $L_{k, C}(x$ and $y$ are tuples of variables ranging in $R$ ). A subset of a power of $R$ that can be defined by a positiveexistential formula is said to be positive-existentially definable. Since the quantifier $\exists$ distributes over $\vee$ (the conjunction or) it is easy to see that finite unions and finite intersections of positive-existential sets are positiveexistential. The positive-existential theory of $R$ in the language $L_{k, R}$ is the set of all positive-existential formulas of $L_{k, C}$ which are true over $R$. It is trivial to see that Question 1.2 is equivalent to the following

QUESTION 1.2(b). Is the positive-existential theory of $R$ in the language $L_{k, C}$ decidable?

We will deal with the case in which $R=F(t)$ is the field of rational functions in the variable $t$, with coefficients in the field $F$. We will assume throughout that $F$ has characteristic zero, so that $\mathbb{Z}$ can be thought of as a subring of $F$. Then Question 1.2 for $R=F(t)$ and $C=\mathbb{Z}[t]$ becomes

QUESTION 1.3. Is the positive-existential theory of $F(t)$ in the language $L_{k, \mathbb{Z}[t]}$ decidable?

In Section 3 we will show that for $k=2$ a negative answer to Question 1.3 follows from [17] for all fields $F$ such that the positive-existential ring theory of $F(t)$, in the language of rings augmented by a symbol for $t$, is undecidable. Such is the case, for example, for $F=\mathbb{R}$, the field of reals (see [2]), so $\mathbf{B q}(2, \mathbb{R}(t), \mathbb{Z}[t])$ has a negative answer. We remark that it is unknown whether the ring theory of $\mathbb{C}(t)$ is undecidable $(\mathbb{C}$ is the field of complex numbers). More accurately, we prove:

TheOREM 1.4. Let $F$ be a field of zero characteristic and let $t$ be a variable. Then multiplication in $F(t)$ is positive-existentially definable in $L_{2, \mathbb{Z}[t]}$. Consequently, if the existential ring theory of $F(t)$ in the language of rings augmented by a symbol for $t$ is undecidable, then the positive-existential

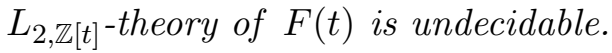

By [2] we obtain:

Corollary 1.5. (a) Assume that $F$ is a real-closed field. Then the subset $\mathbb{Z}$ of $F(t)$ is positive-existentially definable in the language $L_{2, \mathbb{Z}[t]}$ and the positive-existential theory of $F(t)$ in the language $L_{2, \mathbb{Z}[t]}$ is undecidable.

(b) Assume that $F$ is a real field. Then the positive-existential theory of $F(t)$ in the language $L_{2, \mathbb{Z}[t]}$ is undecidable. 
We think it likely that our proof of Theorem 1.4 can be adjusted to any function field of an elliptic curve over $F$ in place of $F(t)$.

For $k \geq 3$ essentially nothing is known on $\mathbf{B q}(k, R, C)$. Our guess is that in some cases at least, if the positive-existential ring theory of $R$ with constants from $C$ is undecidable then the positive-existential $L_{k, C}$-theory of $R$ is undecidable. Our next result is in this direction. In Section 5 we will answer a question similar to Question 1.3, for $k=3$, allowing additional conditions such as " $x \in F$ " and " $x(0)=0$ ", in the case that $R$ is the field $F(t)$ of rational functions in the variable $t$, with coefficients in the field $F$, and for $C=\mathbb{Z}[t]$.

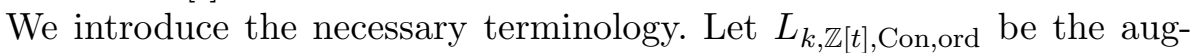
mentation of $L_{k, \mathbb{Z}[t]}$ by the predicate "Con" which is interpreted as

$$
\operatorname{Con}(x) \leftrightarrow x \in F
$$

and by the predicate "ord" which is interpreted as

$$
\operatorname{ord}(x) \leftrightarrow x(0)=0
$$

(the value of the rational function $x$ at $t=0$ is 0 ). The languages $L_{k, \mathbb{Z}[t], \text { Con }}$

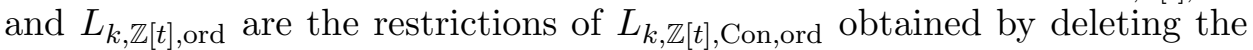
obvious predicate symbols.

We prove:

THEOREM 1.6. Let $F$ be a field of zero characteristic, let $t$ be a variable and let $\xi$ be a primitive cube root of 1 in an extension of $F$. Then

(a) The subset $\mathbb{Z}[\xi] \cap F$ of $F(t)$ is positive-existentially definable in the

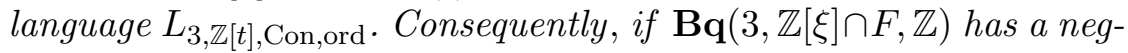
ative answer (for example, if $\mathbb{Z}[\xi] \cap F=\mathbb{Z}$ and $\mathbf{B q}(3)$ has a negative answer) then the positive-existential theory of $F(t)$ in the language $L_{3, \mathbb{Z}[t], \text { Con,ord }}$ is undecidable.

(b) Assume that for some $a, b \in \mathbb{Z}$, with $a b \neq 0, F$ has a subset $D$ such that for all $n \in \mathbb{Z}[\xi] \cap F$ there is a $d \in D$ such that $a n^{3}+b d^{3}=1$. Then the subset $\mathbb{Z}[\xi] \cap F$ of $F(t)$ is positive-existentially definable in the language $L_{3, \mathbb{Z}[t], \text { ord }}$. Hence if $\mathbf{B q}(3, \mathbb{Z}[\xi] \cap F, \mathbb{Z})$ has a negative answer then the positive-existential theory of $F(t)$ in the language $L_{3, \mathbb{Z}[t], \text { ord }}$ is undecidable.

The next corollary provides some examples where the hypothesis of (b) of the Theorem holds.

Corollary 1.7. (a) Assume that $F$ is a field containing the set of algebraic numbers (over $\mathbb{Q}$ ). Then the subset $\mathbb{Z}[\xi]$ of $F(t)$ is positive-existentially definable in the language $L_{3, \mathbb{Z}[t], \text { ord }}$. Hence if $\mathbf{B q}(3, \mathbb{Z}[\xi], \mathbb{Z})$ has a negative answer then the positive-existential theory of $F(t)$ in the language $L_{3, \mathbb{Z}[t], \text { ord }}$ is undecidable. 
(b) Assume that $F$ is a real-closed field. Then the subset $\mathbb{Z}$ of $F(t)$ is positive-existentially definable in the language $L_{3, \mathbb{Z}[t], \text { ord }}$. Hence if $\mathbf{B q}(3)$ has a negative answer then the positive-existential theory of $F(t)$ in the language $L_{3, \mathbb{Z}[t] \text {,ord }}$ is undecidable. In particular, if $\mathbf{B q}(3)$ has a negative answer then the positive-existential theories of $\mathbb{Q}(t)$ and $\mathbb{R}(t)$ in the language $L_{3, \mathbb{Z}[t] \text {,ord }}$ are undecidable.

Both statements are easy consequences of Theorem 1.6: (a) is obvious; (b) follows from Theorem 1.6(b) by taking $a=1=-b$.

We present an outline of the proof of Theorem 1.6 in Section 4 and the complete proof in Section 5.

It is obvious that $\mathbf{B q}(k, R, C)$ is a subproblem of the decidability problem for the positive-existential theory of $R$ with constant symbols for the elements of $C$ (sometimes called "diophantine problem for $(R, C)$ "). Let $L_{t}$ be the language of rings, augmented by the constant-symbol $t$. Undecidability is known for the positive-existential theories in $L_{t}$ of rational function fields $F(t)$ whenever $F$ is a real field or a finite field (see [2], [10] and $[16])$. It is an open problem whether there exists an algebraically closed field $F$ for which the diophantine problem for $(F(t), \mathbb{Z}[t])$ is undecidable. But it is known that the positive-existential theory of any field $F(t)$ in the extension of $L_{t}$ by a predicate for the elements of $F$ and a predicate for "ord $(x)$ " is undecidable (cf. [20]). The question whether these predicates are positive-existentially definable in $L_{t}$ is open, except in some special cases like $F$ the field of real numbers or a finite field. This is the motivation behind our choice to extend the languages $L_{k, \mathbb{Z}[t]}$ by the predicates Con and ord.

For more undecidability results and questions in this direction the reader may consult [4], [13], [19] and the surveys in [11] and [14].

We remark that the method of proof of Theorem 1.6 does not give a positive-existential definition of multiplication in $L_{3, \mathbb{Z}[t], \text { Con,ord }}$. Also the method does not generalize to values of $k$ greater than 3 .

In Section 2 we present a number-theoretical problem, Problem 2.2, which, if answered positively, will imply a negative answer to $\mathbf{B q}(k)$. It is a generalization of the " $n$ squares problem" (or Büchi's problem) of [7], [9] and [17]. Our motivation for presenting it is that if one thinks that it is plausible, then one will consider the undecidability statement of Theorem 1.6 at least as likely.

Throughout, $\mathbb{N}$ is the set of natural numbers $\{1,2, \ldots\}$ and $\mathbb{Z}$ the set of rational integers.

Acknowledgements. The authors would like to thank the referee for his comments. The computations that the referee advised us to carry out in the proof of Lemma 5.1 turned out to be essential in the present form of this lemma. 


\section{The " $n k$ th powers problem"}

Definition 2.1. Let $y=\left(y_{i}\right)_{i=0, \ldots, n-1}$ be a sequence of complex numbers. The difference sequence of $y$ is the sequence $\Delta(y)=(\Delta(y)(i))_{i=0, \ldots, n-2}$ defined by $\Delta(y)(i)=y_{i+1}-y_{i}$. The $\ell$ th difference of $y$, denoted

$$
\Delta^{(\ell)}(y)=\left(\Delta^{(\ell)}(y)(i)\right)_{i=0, \ldots, n-\ell-1},
$$

is defined recursively by $\Delta^{(1)}(y)=\Delta(y)$ and $\Delta^{(\ell+1)}(y)=\Delta\left(\Delta^{(\ell)}(y)\right)$.

Let $k \in \mathbb{Z}, k \geq 2$. Let $R$ be any integral domain of characteristic zero. It is easy to see that for any $x \in R$, the $\ell$ th difference

$$
\Delta^{(\ell)}\left((x+i)_{i=0, \ldots, n-1}^{k}\right)
$$

for $\ell \leq k$ is a sequence of the form

$$
\left(p_{\ell, k}(x), p_{\ell, k}(x+1), \ldots, p_{\ell, k}(x+n-\ell-1)\right)
$$

where $p_{\ell, k}(x)$ is a polynomial in $x$, of degree $k-\ell$, with integer coefficients which depend on $k$ and $\ell$. Observe that $p_{k, k}(x)=k !$.

We now formulate the $n k$ th powers problem (or Büchi's problem for $k$ ).

Problem 2.2. Let $k$ be a rational integer with $k \geq 2$.

(i) Is there a natural number $n \geq k$ such that any sequence of natural numbers $\left(x_{i}\right)_{i=0, \ldots, n-1}$ which satisfies

$$
\Delta^{(k)}\left(\left(x_{i}^{k}\right)_{i=0, \ldots, n-1}\right)=(k !)
$$

(the sequence with $n-k$ terms, each equal to $k !)$ is necessarily a sequence of successive numbers (that is, either $x_{i}=x_{0}+i$ for each $i$, or $x_{i}=x_{0}-i$ for each $\left.i\right)$ ?

(ii) Is there a natural number $n \geq k$ such that any sequence $\left(x_{i}\right)_{i=0, \ldots, n-1}$ of rational numbers which satisfies (2.2.1) is such that $\pm x_{i+1}=$ $\pm x_{i}+1$ for each $i=0, \ldots, n-1$ ? ( the \pm do not have to correspond). Moreover, if $k$ is odd, is it true that, additionally, $x_{i+1}=x_{i}+1$ ?

It is obvious that a positive answer to (ii) of Problem 2.2 implies a positive answer to (i).

For $k=2,(2.2 .1)$ gives a system of $n-2$ equations of the form

$$
x_{i+2}^{2}-2 x_{i+1}^{2}+x_{i}^{2}=2
$$

and for $k=3$ it gives $n-3$ equations of the form

$$
x_{i+3}^{3}-3 x_{i+2}^{3}+3 x_{i+1}^{3}-x_{i}^{3}=6 .
$$

It is obvious from the above observations that if $x_{i+1}=x_{i}+1$ then relation (2.2.1) holds. In fact for $k=2$ more is known.

Lang's Conjecture ([6, Conjecture 5.8]). Let $X$ be a smooth projective algebraic variety of general type, defined over a number field $M$. Then 
there exists a proper Zariski-closed subset $Z$ of $X$ such that for all number fields $K$ containing $M, X(K)-Z(K)$ is finite.

Define $X_{n}$ to be the projective subvariety of $\mathbb{P}^{n}$ cut out by the homogenizations of equations (2.2.1) for $k=2$ (see the first set of equations in Section 3). Vojta proved:

Theorem 2.3 ([17, Theorem 0.5]). If Lang's Conjecture holds for some $X_{n}(\mathbb{Q})$ then the $n 2$ nd powers problem has a positive answer.

In fact Vojta's proof shows that, assuming Lang's Conjecture, equation (2.2.1) for $k=2$ has only the solutions $\pm x_{i+1}+1= \pm x_{i}$ over $\mathbb{Q}$. At this point we have no further evidence in favor of a positive answer to Problem 2.2. In [7] it is shown that a positive answer to the $n 2$ nd powers problem implies a negative answer to $\mathbf{B q}(2)$. We present a similar argument for $k$ th powers, for $k$ odd.

Theorem 2.4. Let $k \geq 3$ be an odd rational integer. If Problem 2.2(ii) has a positive answer then the positive-existential theory of $\mathbb{Z}$ in the language $L_{k, \mathbb{Z}}$ is undecidable, and thus $\mathbf{B q}(k)$ has a negative answer.

Proof. Linear elimination proves the equivalence of the decidability of the positive-existential theory of $\mathbb{Z}$ in the language $L_{k, \mathbb{Z}}$ and $\mathbf{B q}(k)$ (the details are left to the reader).

Assume that $n$ is such that Problem 2.2(ii) (both statements) has a positive answer for $n$. We will represent arbitrary integers as certain linear combinations of $k$ th powers and we will interpret multiplication of two integers in terms of the corresponding representations in a way that is positiveexistential in the language $L_{k, \mathbb{Z}}$. Thus, if the positive-existential theory of $\mathbb{Z}$ in $L_{k, \mathbb{Z}}$ were decidable, then the ring theory of $\mathbb{Z}$ would be decidable, which would contradict the negative answer to Hilbert's tenth problem given in [8].

The formula

$$
\phi\left(y_{0}, \ldots, y_{n-1}\right) \equiv\left[\Delta^{(k)}\left(\left(y_{i}\right)_{i=0, \ldots, n-1}\right)=(k !)\right] \bigwedge_{i=0, \ldots, n} \text { " } y_{i} \text { is a } k \text { th power" }
$$

is a formula of the language $L_{k, \mathbb{Z}}$. Having assumed a positive answer to Problem 2.2(ii) we find that $\phi\left(y_{0}, \ldots, y_{n-1}\right)$ implies that, setting $y_{i}=x_{i}^{k}$, we have

$$
x_{i+1}=x_{i}+1 .
$$

Then, obviously, writing $x=x_{0}$, we have

$$
y_{i+1}-y_{i}=p_{1, k}(x+i) .
$$

It is easy to see that

$$
\left\{X^{k},(X+1)^{k}, \ldots,(X+k)^{k}\right\}
$$


is a basis of the vector space of polynomials in the variable $X$ of degree at most $k$ over $\mathbb{Q}$. Hence both $X$ and $X^{2}$ can be written as $\mathbb{Q}$-linear combinations of elements of this basis, say

$$
X=\sum_{i} c_{i}(X+i)^{k} \quad \text { and } \quad X^{2}=\sum_{i} d_{i}(X+i)^{k}
$$

for some fixed rational numbers (depending on $k$ ) $c_{i}$ and $d_{i}$. Write

$$
h_{1}\left(Y_{0}, \ldots, Y_{k}\right)=\sum_{i} c_{i} Y_{i} \text { and } h_{2}\left(Y_{0}, \ldots, Y_{k}\right)=\sum_{i} d_{i} Y_{i} .
$$

We interpret arbitrary elements $x$ of $\mathbb{Z}$ as the quantities $x=h_{1}\left(y_{0}, \ldots, y_{k}\right)$ for which

$$
\exists y_{k+1}, \ldots, y_{n-1} \phi\left(y_{0}, \ldots, y_{k}, \ldots, y_{n}\right)
$$

is true. Then we have

$$
x^{2}=h_{2}\left(y_{0}, \ldots, y_{k}\right),
$$

hence we obtain a representation of the graph of the squaring function in $L_{k, \mathbb{Z}}$ (in the end we will need to clear denominators of terms of the equations so that only integers appear as coefficients). Finally, we interpret multiplication using the equivalence

$$
c=a b \leftrightarrow(a+b)^{2}=a^{2}+b^{2}+2 c .
$$

REMARK 2.5. If the $n k$ th powers problem has a positive answer over $\mathbb{Q}$ then one obtains a result similar to that of Theorem 2.4 for $\mathbb{Q}$. But undecidability does not follow from current knowledge: the analogue of Hilbert's tenth problem for $\mathbb{Q}$ is an open problem (cf. [11]).

REMARK 2.6. It seems plausible that the " $n k$ th powers problem" may have a positive answer over any ring of integers of a number field, or in any number field. Certainly it has a negative answer in any extension of the ring of real algebraic integers. We cannot predict a characterization of the extensions of $\mathbb{Z}$ where it holds.

3. Systems of squares. We consider Question 1.3 for $k=2$ (that is, $\mathbf{B q}(2, F(t), \mathbb{Z}[t])$ of the Introduction) where $F$ is a field of characteristic zero. In what follows $X_{n}$ is the projective subvariety of the projective $n$-space $\mathbb{P}^{n}$, over $\mathbb{C}$, cut out by the equations (in projective coordinates $\left(x, x_{1}, \ldots, x_{n}\right)$ )

$$
x_{i}^{2}+x_{i-2}^{2}=2 x_{i-1}^{2}+2 x^{2}, \quad i=3, \ldots, n .
$$

In [17] P. Vojta observed that

TheORem 3.1. For $n \geq 6$ the variety $X_{n}$ is a surface of general type.

Then he showed 
Theorem 3.2 ([17, Theorem 3.1]). For $n \geq 8$, the only curves on $X_{n}$ of geometric genus 0 or 1 are the "trivial" lines

$$
\pm x_{i}= \pm x_{1}-(i-1) x, \quad i=2, \ldots, n .
$$

This has as an immediate consequence the following:

Corollary 3.3. Let $n \geq 8$. Assume that $F$ is a field of zero characteristic and that $y_{1}, \ldots, y_{n} \in F(t)$ are not all constant (i.e. in $F$ ) and satisfy

$$
y_{i}^{2}+y_{i-2}^{2}=2 y_{i-1}^{2}+2
$$

for $i=3, \ldots, n$. Then for some $\varepsilon= \pm 1$ and for all $i=2, \ldots, n$ we have

$$
\pm y_{i}=\varepsilon y_{1}-(i-1) .
$$

Proof. Assume that the $y_{i}$ are as in the hypothesis. They involve finitely many coefficients. Embed the subring of $F$ generated by those coefficients into the field of complex numbers and observe that the hypothesis of the corollary remains true with $F$ replaced by $\mathbb{C}$. So, without loss of generality, we assume that $F=\mathbb{C}$. Write $y_{i}=a_{i} / b$ with $a_{i}, b \in F[t]$ such that the greatest common divisor of the elements of the set $\left(b, a_{1}, \ldots, a_{n}\right)$ is the unit ideal. Homogenize simultaneously all the $a_{i}$ and $b$, that is, substitute $t$ by $t_{1} / t_{0}$, and find homogeneous polynomials $B\left(t_{0}, t_{1}\right), A_{1}\left(t_{0}, t_{1}\right), \ldots, A_{n}\left(t_{0}, t_{1}\right)$ of the form

$$
B\left(t_{0}, t_{1}\right)=t_{0}^{r} b\left(\frac{t_{1}}{t_{0}}\right) \quad \text { and } \quad A_{i}\left(t_{0}, t_{1}\right)=t_{0}^{r_{i}} a_{i}\left(\frac{t_{1}}{t_{0}}\right)
$$

so that the only common zero of all $B$ and $A_{i}$ is $\left(t_{0}, t_{1}\right)=(0,0)$. Then the correspondence

$$
\left(t_{0}, t_{1}\right) \mapsto\left(B\left(t_{0}, t_{1}\right), A_{1}\left(t_{0}, t_{1}\right), \ldots, A_{n}\left(t_{0}, t_{1}\right)\right)
$$

is a map from the projective line $\mathbb{P}(\mathbb{C})$ into $X_{n}$. By Hurwitz's formula (cf. [3]) the image of that map is a projective curve of geometric genus 0 , or, in other words,

$$
\left(x, x_{1}, \ldots, x_{n}\right)=\left(B\left(t_{0}, t_{1}\right), A_{1}\left(t_{0}, t_{1}\right), \ldots, A_{n}\left(t_{0}, t_{1}\right)\right)
$$

is a parametrization of a curve on $X_{n}$ of geometric genus 0 . Hence, by Theorem 3.2, we have

$$
\pm x_{i}= \pm x_{1}-(i-1) x .
$$

The latter relation implies that for each $i=2, \ldots, n$ we have

$$
\pm y_{i}= \pm y_{1}-(i-1) .
$$

Finally, we show that the \pm correspond in the way stated in the conclusion: Set $\pm y_{2}=\varepsilon y_{1}-1$ for some $\varepsilon= \pm 1$ and assume that for some $k \geq 2$ we have $\pm y_{i}=\varepsilon y_{1}-(i-1)$ for $i=2, \ldots, k$ but $\pm y_{k+1}=-\varepsilon y_{1}-k$. Then

$$
y_{k+1}^{2}+y_{k-1}^{2}-2 y_{k}^{2}=2 \varepsilon(k+1) y_{1}+2 .
$$


On the other hand, by hypothesis, $\left(y_{k-1}, y_{k}, y_{k+1}\right)$ satisfies

$$
y_{k+1}^{2}+y_{k-1}^{2}-2 y_{k}^{2}=2 .
$$

Equating the right hand sides of the last two equalities we obtain

$$
\varepsilon(k+1) y_{1}=0 .
$$

Then all $y_{i}$ are in $F$, which contradicts the hypothesis. The conclusion follows.

Note. Corollary 3.3 also follows from Theorem 0.6 of [17] which states: Let $n \geq 8$ be an integer, and let $f: \mathbb{C} \rightarrow X_{n}$ be a non-constant holomorphic map. Then the image of $f$ lies in one of the "trivial lines".

The proof of this theorem is by results of Vojta in Nevanlinna theory while the proof of Theorem 3.2 is based on algebro-geometric tools.

The proof of Corollary 3.3 is the following (the authors thank Paul Vojta for pointing this out): As in the above proof, assume without loss of generality that $F=\mathbb{C}$. Then the array $\left(y_{1}, \ldots, y_{n}\right)$ of rational functions induces a meromorphic map from $\mathbb{C}$ to $X_{n}$, which extends to a holomorphic map from $\mathbb{C}$ to $X_{n}$, by the valuative criterion of properness (for the terminology and the necessary facts see [18]). Then the latter theorem implies the conclusion.

Proof of Theorem 1.4. Let $\phi(z, w)$ denote the formula

$$
\begin{aligned}
& \exists w_{1}, \ldots, w_{8} \in F(t)[w= w_{1} \wedge 2 z=w_{2}-w_{1}-1 \\
&\left.\bigwedge_{i=3, \ldots, 8} w_{i}+w_{i-2}=2 w_{i-1}+2 \bigwedge_{i=1, \ldots, 8} P_{2}\left(w_{i}\right)\right] .
\end{aligned}
$$

Assume that $w=z^{2}$. Then it is trivial to see that $\phi(z, w)$ holds true by taking $w_{i+1}=(z+i)^{2}$ for $i=2, \ldots, 7$. Now assume that $\phi(z, w)$ is true. We claim that then either $w=z^{2}$ or $w \in F$. Assume that $w \notin F$. Let $w_{i}$ satisfy the quantifier-free part of $\phi$. Set $w_{i}=y_{i}^{2}$ for some $y_{i} \in F(t)$ (since $\phi$ is true such $y_{i}$ exist). Then $y_{1} \notin F$ and by Corollary 3.3 , for some $\varepsilon= \pm 1$ and for all $i=2, \ldots, n$ we have

$$
\pm y_{i}=\varepsilon y_{1}-(i-1)
$$

Then $w=y_{1}^{2}$ and

$$
2 z=\left(\varepsilon y_{1}-1\right)^{2}-y_{1}^{2}-1,
$$

hence $z=-\varepsilon y_{1}$ and $w=z^{2}$.

It is then trivial to see that $w=z^{2}$ is equivalent to $\phi(z, w) \wedge \phi\left(t z, t^{2} w\right) \wedge \phi\left(z+t, w+2 t z+t^{2}\right) \wedge \phi\left(t(z+t), t^{2}\left(w+2 t z+t^{2}\right)\right)$.

Thus squaring, and hence multiplication, is definable in $L_{2, \mathbb{Z}[t]}$. 
It follows that for any field $F$ of characteristic zero the positive-existential $L_{2, \mathbb{Z}[t]}$ theory of $F(t)$ is decidable if and only if the existential ring theory (in the language $L_{t}$ of the Introduction) of $F(t)$ is decidable.

\section{The case $k=3$ for fields of rational functions: Outline of} method. We consider the case $k=3$. Let $\xi$ be a primitive cube root of unity. Consider the following equation:

$$
\left(1-t^{3}\right) y^{3}=1-x^{3}
$$

over $F(t)$. The crucial fact for the proof of Theorem 1.6 is that for any solution $(x, y)$ of (MD1), the value of $y$ at $t=1$ is in $\mathbb{Z}[\xi]$. This results from the following analysis. Consider the elliptic curve $\mathcal{E}$ defined by the affine equation

$$
X^{3}+Y^{3}=1
$$

(for the theory of elliptic curves the reader may consult [5] and [15]). It is well known that $\mathcal{E}$ together with any point $\mathcal{O}$ on the line at infinity is an elliptic curve over $F$. Fix an $s$ such that $t^{3}+s^{3}=1$. For each $x, y \in F(t)$ which satisfy (MD1) the rational function

$$
(t, s) \mapsto(x(t), s y(t))
$$

defines a function from $\mathcal{E}$ into itself. By a theorem of Weil, any such function is the translation (by some point of $\mathcal{E}$, rational over $F$ ) of an endomorphism of $\mathcal{E}$. We will show that in our situation the set of possible translations is finite. So, modulo (in the group sense) a finite set, one can associate to each solution of (MD1) an endomorphism of $\mathcal{E}$. The ring of endomorphisms of $\mathcal{E}$ is isomorphic to $\mathbb{Z}[\xi]$. It turns out that, depending on the endomorphism $[n]$, three cases can occur: the function $[n](t, s)$ can be of the form $\left(x_{n}, s y_{n}\right)$, $\left(s x_{n}, y_{n}\right)$ or $\left(\frac{1}{s} x_{n}, \frac{1}{s} y_{n}\right)$. Each of these cases gives an equation which is either (MD1) or one of two similar equations ((MD2) and (MD0) of the next section). Conversely, the rational maps which are defined by any of those equations form a subset of the group $\operatorname{Rat}_{F}(\mathcal{E})$ of rational maps from $\mathcal{E}$ to $\mathcal{E}$ over $F$. We will prove that this subset is actually a subgroup which is isomorphic to the group $\operatorname{End}_{F}(\mathcal{E}) \oplus \mathcal{E}_{3}(F)$, where $\operatorname{End}_{F}(\mathcal{E})$ denotes the ring of endomorphisms on $\mathcal{E}$, and $\mathcal{E}_{3}(F)$ the group of rational points of order 3 on $\mathcal{E}$. Finally, we will show that any $n \in \mathbb{Z}[\xi]$ is the value of a rational function (such as $y$ ) associated to some solution of one of the equations (MDi), at $t=1$. Thus we will obtain a definition of $F \cap \mathbb{Z}[\xi]$ over $F(t)$ which

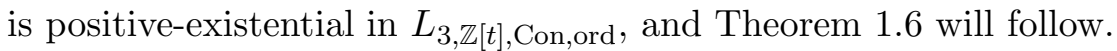

We note that elliptic curves of the form of equations (MDi) have been studied first by Yu. Manin and J. Denef. 
5. Systems of cubes. Throughout this section $F$ is a field of characteristic zero. We consider the elliptic curve $\mathcal{E}$ defined by the projective equation

$$
X^{3}+Y^{3}=Z^{3},
$$

with the distinguished point being $\mathcal{O}=[1,-1,0]$ on the "line at infinity" $Z=0$ (equations of this type have been studied in detail in [12]). Note that there are two other points on the line at infinity, that is, $[1,-\xi, 0]$ and $[-\xi, 1,0]$, where $\xi \neq 1$ denotes a cube root of unity. The curve $\mathcal{E}$ has complex multiplication, and its $j$-invariant is 0 (see [15, Chapter III, Exercise 3.3, p. 104]). Therefore it has six automorphisms (see [15, Chapter III, Theorem 10.1, p. 103]). Writing

$$
x=\frac{X}{Z} \quad \text { and } \quad y=\frac{Y}{Z}
$$

we obtain the equation

$$
x^{3}+y^{3}=1
$$

which defines the affine part $\mathcal{E}_{a}$ of the elliptic curve $\mathcal{E}$. The six automorphisms are given by $[1],[\xi],\left[\xi^{2}\right]$ and their negatives, where $[\xi]$ and $\left[\xi^{2}\right]$ are defined by

$$
[\xi](x, y)=(\xi x, \xi y) \text { and }\left[\xi^{2}\right](x, y)=\left(\xi^{2} x, \xi^{2} y\right) .
$$

We will now describe the addition law on $\mathcal{E}$. If $P=\left(x_{0}, y_{0}\right)$ its negative is given by

$$
\ominus P=\left(y_{0}, x_{0}\right) .
$$

Let $P_{i}=\left(x_{i}, y_{i}\right), i=1,2$, be two points on $\mathcal{E}$. If

$$
\left(x_{3}, y_{3}\right)=\left(x_{1}, y_{1}\right) \oplus\left(x_{2}, y_{2}\right)
$$

then we find, by applying the method described in [15, Chapter III, $\S 2$, pp. 55-59],

$$
y_{3}=\frac{-3 \lambda^{2} \nu}{1+\lambda^{3}}-x_{1}-x_{2},
$$

which, if $x_{1} x_{2} \neq 0$, can be written

$$
y_{3}=\frac{1-\nu^{3}}{\left(1+\lambda^{3}\right) x_{1} x_{2}}
$$

where $\lambda$ and $\nu$ are given, if $x_{1} \neq x_{2}$, by

$$
\lambda=\frac{y_{2}-y_{1}}{x_{2}-x_{1}} \quad \text { and } \quad \nu=\frac{y_{1} x_{2}-y_{2} x_{1}}{x_{2}-x_{1}}
$$

and if $x_{1}=x_{2}=x$, by

$$
\lambda=-\frac{x^{2}}{y^{2}} \quad \text { and } \quad \nu=\frac{1}{y^{2}} .
$$

The first coordinate $x_{3}$ is then given by $x_{3}=\lambda y_{3}+\nu$. But by symmetry, $x_{3}$ can also be obtained by exchanging $x_{1}$ with $y_{1}$, and $x_{2}$ with $y_{2}$, in $y_{3}$. In 
particular, we obtain the "duplication formula" for the curve $\mathcal{E}$ :

$$
2(x, y)=\left(y \frac{x^{3}+1}{y^{3}-x^{3}}, x \frac{y^{3}+1}{x^{3}-y^{3}}\right)=\left(y \frac{x^{3}+1}{1-2 x^{3}}, x \frac{x^{3}-2}{1-2 x^{3}}\right) .
$$

In order to compute the order of the points at infinity, it is convenient to write the formula also in projective coordinates:

$$
2[T, S, R]=\left[S\left(T^{3}+R^{3}\right), T\left(T^{3}-2 R^{3}\right), R\left(R^{3}-2 T^{3}\right)\right] .
$$

We find

$$
2[1,-\xi, 0]=[-\xi, 1,0]=\ominus[1,-\xi, 0],
$$

which implies that the points $[1,-\xi, 0]$, as well as $[-\xi, 1,0]$, are of order 3 . We observe that the point $[1,0,1]$ is also of order 3 , since

$$
2[1,0,1]=[0,1,1]=\ominus[1,0,1] .
$$

The images of this point under each of the six automorphisms give six new points of order 3 . So we found all the nine points of order 3 on $\mathcal{E}$, remembering that three of them (counting the neutral) are on the line at infinity (see [15, Chapter 3, Corollary 6.4, p. 89]).

More generally, the addition formula is given in projective coordinates by the following. If

$$
\left[X_{1}, Y_{1}, Z_{1}\right] \oplus\left[X_{2}, Y_{2}, Z_{2}\right]=\left[X_{3}, Y_{3}, Z_{3}\right]
$$

then we can choose:

$$
\begin{aligned}
X_{3} & =Z_{1} Z_{2}\left(Z_{2} Y_{1}-Z_{1} Y_{2}\right)+X_{1} X_{2}\left(X_{1} Y_{2}-X_{2} Y_{1}\right), \\
Y_{3} & =Z_{1} Z_{2}\left(Z_{2} X_{1}-Z_{1} X_{2}\right)+Y_{1} Y_{2}\left(X_{2} Y_{1}-X_{1} Y_{2}\right), \\
Z_{3} & =X_{1} X_{2}\left(X_{1} Z_{2}-X_{2} Z_{1}\right)+Y_{1} Y_{2}\left(Y_{1} Z_{2}-Y_{2} Z_{1}\right) .
\end{aligned}
$$

We deduce from this the "triplication formula" (note that by applying the addition formula to $2(T, S, R) \oplus(T, S, R)$, all the coordinates $X_{3}, Y_{3}$ and $Z_{3}$ have $T+S$ as a common factor, and this simplifies much the computation): $3[T, S, R]=\left[-T^{9}-3 R^{3} T^{6}+6 R^{6} T^{3}-R^{9}\right.$,

$$
\left.T^{9}-6 R^{3} T^{6}+3 R^{6} T^{3}+R^{9}, T S R\left(3 T^{6}-3 R^{3} T^{3}+3 R^{6}\right)\right] .
$$

If $n=n_{1}+n_{2} \xi \in \mathbb{Z}[\xi]$ and $i \in\{0,1,2\}$, we will write $n \sim i$ if $n_{1}+n_{2}$ is congruent to $i \bmod 3$ (this corresponds to congruence modulo $1-\xi$ ).

Lemma 5.1. (i) Let $S, T, R$ be such that $S^{3}+T^{3}=R^{3}$. For any $n \in \mathbb{Z}[\xi]$, there exist homogeneous polynomials $F_{n}, G_{n}, H_{n}$ in $F\left[T^{3}, R^{3}\right]$ such that

$$
n[T, S, R]= \begin{cases}{\left[F_{n}, G_{n}, T S R H_{n}\right]} & \text { if } n \sim 0, \\ {\left[T F_{n}, S G_{n}, R H_{n}\right]} & \text { if } n \sim 1, \\ {\left[S F_{n}, T G_{n}, R H_{n}\right]} & \text { if } n \sim 2 .\end{cases}
$$


(ii) For any $n \in \mathbb{Z}[\xi]$, the three coordinates of $n[T, S, R]$ have the same global degree $d_{n}$ in the variables $T, S, R$ (not uniquely determined).

(iii) Let $X, Y$ and $Z$ be elements of $F(T, R)$. Then $(X, Y, S Z) \oplus(T, S, R)$ is of the form $\left(X_{0}, S Y_{0}, Z_{0}\right),(X, S Y, Z) \oplus(T, S, R)$ is of the form $\left(S X_{1}, Y_{1}, Z_{1}\right)$, and $(S X, Y, Z) \oplus(T, S, R)$ is of the form $\left(X_{2}, Y_{2}, S Z_{2}\right)$, for some functions $X_{i}, Y_{i}$ and $Z_{i}$ in $F(T, R)$.

(iv) Write $s=S / R$ and $t=T / R$ so that $s^{3}+t^{3}=1$. For any $n \in \mathbb{Z}[\xi]$, there exist homogeneous polynomials $X_{n}, Y_{n}, Z_{n}$ in $F[T, R]$, and, if $n \neq 0$, rational functions $x_{n}, y_{n}$ in $F(t)$ such that

$$
n[T, S, R]= \begin{cases}{\left[X_{n}, Y_{n}, S Z_{n}\right]} & \text { if } n \sim 0, \\ {\left[X_{n}, S Y_{n}, Z_{n}\right]} & \text { if } n \sim 1, \\ {\left[S X_{n}, Y_{n}, Z_{n}\right]} & \text { if } n \sim 2,\end{cases}
$$

and, on the affine part of $\mathcal{E}$,

$$
n(t, s)= \begin{cases}\left(\frac{1}{s} x_{n}, \frac{1}{s} y_{n}\right) & \text { if } n \sim 0, \\ \left(x_{n}, s y_{n}\right) & \text { if } n \sim 1, \\ \left(s x_{n}, y_{n}\right) & \text { if } n \sim 2 .\end{cases}
$$

(v) We have

$$
x_{n+a}(1)=\frac{y_{n}(1)}{a} \frac{1}{y_{n}^{2}(1)-a x_{n}(1)}
$$

for all $n \sim 2$.

Proof. We prove (i) by induction in three steps. Observe that the assertion is true for $n=0,1,2,3$. Let $a=1$ or $\xi$.

1. First suppose that $n \sim 1$. We apply the addition formula to

$$
\left[T F_{n}, S G_{n}, R H_{n}\right] \oplus[a T, a S, R]
$$

and find polynomials $U, V, W$ such that

$$
\begin{aligned}
U & =S\left[R^{3} H_{n}\left(G_{n}-a H_{n}\right)+a^{2} T^{3} F_{n}\left(F_{n}-G_{n}\right)\right], \\
V & =T\left[R^{3} H_{n}\left(F_{n}-a H_{n}\right)+a^{2} S^{3} G_{n}\left(G_{n}-F_{n}\right)\right], \\
W & =R\left[T^{3} F_{n}\left(a F_{n}-a^{2} H_{n}\right)+S^{3} G_{n}\left(a G_{n}-a^{2} H_{n}\right)\right] .
\end{aligned}
$$

Choose $F_{n+a}=U / S, G_{n+a}=V / T$ and $H_{n+a}=W / R$.

2 . Now suppose that $n \sim 2$. We apply the addition formula to

$$
\left[S F_{n}, T G_{n}, R H_{n}\right] \oplus[a T, a S, R]
$$

and find polynomials

$$
\begin{aligned}
U & =T\left(R^{3} G_{n} H_{n}+a^{2} S^{3} F_{n}^{2}\right)-S\left(a R^{3} H_{n}^{2}+a^{2} T^{3} F_{n} G_{n}\right), \\
V & =S\left(R^{3} F_{n} H_{n}+a^{2} T^{3} G_{n}^{2}\right)-T\left(a R^{3} H_{n}^{2}+a^{2} S^{3} F_{n} G_{n}\right), \\
W & =a T S R\left[S\left(F_{n}^{2}-a G_{n} H_{n}\right)+T\left(G_{n}^{2}-a F_{n} H_{n}\right)\right] .
\end{aligned}
$$


In the formula for $W$, write $A=F_{n}^{2}-a G_{n} H_{n}$ and $B=G_{n}^{2}-a F_{n} H_{n}$. So we have $W=a T S R[S A+T B]$. Note that

$$
(S A+T B)\left(S^{2} A^{2}-S T A B+T^{2} B^{2}\right)=S^{3} A^{3}+T^{3} B^{3} .
$$

By multiplying $U, V$ and $W$ by $S^{2} A^{2}-S T A B+T^{2} B^{2}$, and writing the new quantities $X_{n+1}, Y_{n+1}$ and $Z_{n+1}$ respectively, we obtain new polynomials $U_{1}, V_{1}$ and $W_{1}$. The polynomials $U_{1}$ and $V_{1}$ can be written in the form

$$
S^{3} \alpha+S^{2} T \beta+S T^{2} \gamma+T^{3} \delta
$$

It happens that in both cases the polynomials $\beta$ and $\gamma$ are 0 . The computation finally gives:

$$
\begin{aligned}
U_{1}= & T^{3}\left(R^{3} G_{n} H_{n}+a^{2} S^{3} F_{n}^{2}\right)\left(G_{n}^{2}-a F_{n} H_{n}\right)^{2} \\
& -S^{3}\left(a R^{3} H_{n}^{2}+a^{2} T^{3} F_{n} G_{n}\right)\left(F_{n}^{2}-a G_{n} H_{n}\right)^{2}, \\
V_{1}= & S^{3}\left(R^{3} F_{n} H_{n}+a^{2} T^{3} G_{n}^{2}\right)\left(F_{n}^{2}-a G_{n} H_{n}\right)^{2} \\
& -T^{3}\left(a R^{3} H_{n}^{2}+a^{2} S^{3} F_{n} G_{n}\right)\left(G_{n}^{2}-a F_{n} H_{n}\right)^{2}, \\
W_{1}= & a T S R\left[S^{3}\left(F_{n}^{2}-a G_{n} H_{n}\right)^{3}+T^{3}\left(G_{n}^{2}-a F_{n} H_{n}\right)^{3}\right] .
\end{aligned}
$$

Choose $F_{n+a}=U_{1}, G_{n+a}=V_{1}$ and $H_{n+a}=W_{1} / T S R$.

3. Suppose finally that $n \sim 0$ and apply the addition formula to

$$
\left[F_{n}, G_{n}, T S R H_{n}\right] \oplus[a T, a S, R] .
$$

We find polynomials

$$
\begin{aligned}
U & =T\left[S\left(R^{3} G_{n} H_{n}+a^{2} F_{n}^{2}\right)-T\left(a R^{3} S^{3} H_{n}^{2}+a^{2} F_{n} G_{n}\right)\right], \\
V & =S\left[T\left(R^{3} F_{n} H_{n}+a^{2} G_{n}^{2}\right)-S\left(a R^{3} T^{3} H_{n}^{2}+a^{2} F_{n} G_{n}\right)\right], \\
W & =a R\left[T\left(F_{n}^{2}-a S^{3} G_{n} H_{n}\right)+S\left(G_{n}^{2}-a T^{3} F_{n} H_{n}\right)\right] .
\end{aligned}
$$

We use the same technique as in the second step to obtain

$$
\begin{aligned}
U_{1}= & T\left[S^{3}\left(R^{3} G_{n} H_{n}+a^{2} F_{n}^{2}\right)\left(G_{n}^{2}-a T^{3} F_{n} H_{n}\right)^{2}\right. \\
& \left.-T^{3}\left(a R^{3} S^{3} H_{n}^{2}+a^{2} F_{n} G_{n}\right)\left(F_{n}^{2}-a S^{3} G_{n} H_{n}\right)^{2}\right], \\
V_{1}= & S\left[T^{3}\left(R^{3} F_{n} H_{n}+a^{2} G_{n}^{2}\right)\left(F_{n}^{2}-a S^{3} G_{n} H_{n}\right)^{2}\right. \\
& \left.-S^{3}\left(a R^{3} T^{3} H_{n}^{2}+a^{2} F_{n} G_{n}\right)\left(G_{n}^{2}-a T^{3} F_{n} H_{n}\right)^{2}\right], \\
W_{1}= & a R\left[T^{3}\left(F_{n}^{2}-a S^{3} G_{n} H_{n}\right)^{3}+S^{3}\left(G_{n}^{2}-a T^{3} F_{n} H_{n}\right)^{3}\right] .
\end{aligned}
$$

Choose $F_{n+a}=U_{1} / T, G_{n+a}=V_{1} / S$ and $H_{n+a}=W_{1} / R$.

Note that we proved the first part of the lemma for all integers $n_{1}+n_{2} \xi \in$ $\mathbb{Z}[\xi]$ such that $n_{1}$ and $n_{2}$ are non-negative. It follows obviously for all the other integers in $\mathbb{Z}[\xi]$. The details are left to the reader.

(ii) and (iii) are immediate consequences of the computations above.

(iv) The first part is a direct consequence of (i). The second part is a consequence of (ii). The fact that $d_{n}$ is not uniquely determined by $n$ (since we are in projective coordinates) does not matter: we divide each coordinate 
of $n[T, S, R]$ by $R^{d_{n}}$ in order to obtain new coordinates in the variables $t$ and $s$. The conclusion follows.

(v) Consider the second step of the proof of (i). In $U_{1}$ and $(1 / S) W_{1}$, replace $T$ and $R$ by $1, F_{n}$ by $x_{n}(1), G_{n}$ by $y_{n}(1)$ and $H_{n}$ by 1 . Observe that $S^{3}=R^{3}-T^{3}$ must be replaced by 0 . We get

$$
x_{n+a}(1)=\frac{y_{n}(1)}{a} \frac{\left(y_{n}^{2}(1)-a x_{n}(1)\right)^{2}}{\left(y_{n}^{2}(1)-a x_{n}(1)\right)^{3}}=\frac{y_{n}(1)}{a} \frac{1}{y_{n}^{2}(1)-a x_{n}(1)} .
$$

Lemma 5.2. For any $[n] \in \operatorname{End}_{F}(\mathcal{E}), n \neq 0$, we have

$$
x_{n}=y_{-n} .
$$

Proof. First consider $n \sim 1$. Therefore $-n \sim 2$. We have

$$
\left(s x_{-n}, y_{-n}\right)=[-n](t, s)=\ominus[n](t, s)=\ominus\left(x_{n}, s y_{n}\right)=\left(s y_{n}, x_{n}\right)
$$

using Lemma 5.1(iv). And we have

$$
\left(\frac{1}{s} x_{-n}, \frac{1}{s} y_{-n}\right)=[-n](t, s)=\ominus[n](t, s)=\ominus\left(\frac{1}{s} x_{n}, \frac{1}{s} y_{n}\right)=\left(\frac{1}{s} y_{n}, \frac{1}{s} x_{n}\right)
$$

for $n \sim 0$.

Denote by $\operatorname{Rat}_{F}(\mathcal{E})$ the group of $F$-rational maps $\mathcal{E} \rightarrow \mathcal{E}$, by $\operatorname{End}_{F}(\mathcal{E})$ the ring of endomorphisms of $\mathcal{E}$, and by $\mathcal{E}(F)$ the group of $F$-rational points of $\mathcal{E}$. Write

$$
\begin{aligned}
& \left.R_{0}=\left\{f \in \operatorname{Rat}_{F}(\mathcal{E}) \mid \exists X, Y, Z \in F[T, R], f([T, S, R])=[X, Y, S Z]\right)\right\}, \\
& \left.R_{1}=\left\{f \in \operatorname{Rat}_{F}(\mathcal{E}) \mid \exists X, Y, Z \in F[T, R], f([T, S, R])=[X, S Y, Z]\right)\right\}, \\
& \left.R_{2}=\left\{f \in \operatorname{Rat}_{F}(\mathcal{E}) \mid \exists X, Y, Z \in F[T, R], f([T, S, R])=[S X, Y, Z]\right)\right\} .
\end{aligned}
$$

We will identify $[T, S, R]$ with the identity map in $\operatorname{Rat}_{F}(\mathcal{E})$. Also we will use the symbol $\oplus$ for the addition $\operatorname{in}_{\operatorname{Rat}_{F}}(\mathcal{E})$.

Lemma 5.3. Let $i \in\{0,1,2\}$. Denote by $\bar{i}$ the congruence class of $i$ mod 3. Then

$$
R_{i} \oplus[T, S, R]=R_{\overline{i+1}}
$$

and the union $\bigcup_{i=0}^{2} R_{i}$ is a subgroup of $\operatorname{Rat}_{F}(\mathcal{E})$.

Proof. From Lemma 5.1(iii), we know that $R_{i} \oplus[T, S, R] \subset R_{\overline{i+1}}$. Actually this inclusion is an equality of sets:

$$
R_{0} \subset R_{1} \ominus[T, S, R] \subset R_{2} \ominus 2[T, S, R] \subset R_{0} \ominus 3[T, S, R]=R_{0} .
$$

The last equality comes from the fact that $3[T, S, R] \in R_{0}$. So we have

$$
\bigcup_{i=0}^{2} R_{i}=R_{0} \cup\left(R_{0} \oplus[T, S, R]\right) \cup\left(R_{0} \oplus 2[T, S, R]\right) .
$$

Therefore it suffices to prove that $R_{0}$ is a subgroup of $\operatorname{Rat}_{F}(\mathcal{E})$. But this is obvious from the addition formula. 
We consider the natural morphism of groups, which is an isomorphism (see [15, Chapter III, §4, p. 75]):

$$
\Psi: \operatorname{End}_{F}(\mathcal{E}) \oplus \mathcal{E}(F) \rightarrow \operatorname{Rat}_{F}(\mathcal{E}) .
$$

There are actually two natural ways to define $\Psi(P)$ if $P \in \mathcal{E}(F)$. We can define it as the translation map by $P$ or as the constant map. We will choose the second way. Write $\mathcal{E}_{3}(F)$ for the set of points of order 3 of the curve $\mathcal{E}$ (including the neutral). Write

$$
P_{1}^{a}=[a, 0,1], \quad P_{2}^{a}=[0, a, 1], \quad P_{0}^{a}=[1,-a, 0],
$$

where $a$ denotes any of the three cube roots of unity. Write

$$
P_{i}=\left\{P_{i}^{a} \mid a=1, \xi, \xi^{2}\right\} .
$$

With the following lemma one can see how a point on $\mathcal{E}$ behaves after adding a point of order 3 .

Lemma 5.4. Let $[U, V, W]$ be a point on $\mathcal{E}$, and $a=1, \xi$ or $\xi^{2}$. Then

$$
\begin{aligned}
{[U, V, W] \oplus[1,-a, 0] } & =\left[a U, a^{2} V, W\right], \\
{[U, V, W] \oplus[0, a, 1] } & =\left[-a W, U,-a^{2} V\right], \\
{[U, V, W] \oplus[a, 0,1] } & =\left[V,-a W,-a^{2} U\right] .
\end{aligned}
$$

Proof. To get the first and the second equalities we apply the addition formula and multiply the three coordinates of the results respectively by

$$
\frac{U^{2}-a^{2} U V+a V^{2}}{W^{3}}
$$

and

$$
\frac{W^{2}+a^{2} V W+a V^{2}}{U^{3}} .
$$

In order to find the third equality, observe that $[a, 0,1]$ is the negative of $[0, a, 1]$ and use the second equality.

If one does not like using the addition formula, one could observe that the right hand sides of the equalities define morphisms without fixed points, hence translations; the images of the origin under these translations give the constants on the left hand sides.

Write

$$
U_{i}=\left\{[n] \oplus P_{j}^{a} \mid[n] \in \operatorname{End}_{F}(\mathcal{E}), n+j \sim i \text { and } a=1, \xi \text { or } \xi^{2}\right\} .
$$

Lemma 5.5. We have

$$
\Psi^{-1}\left(\bigcup_{i=0}^{2} R_{i}\right)=\operatorname{End}_{F}(\mathcal{E}) \oplus \mathcal{E}_{3}(F) .
$$

More precisely, $\Psi^{-1}\left(R_{i}\right)=U_{i}$. 
Proof. It is clear from Lemma 5.1(i) that

$$
\Psi^{-1}\left(\bigcup_{i=0}^{2} R_{i}\right) \supseteq \operatorname{End}_{F}(\mathcal{E}) \text { and } \Psi^{-1}\left(\bigcup_{i=0}^{2} R_{i}\right) \supseteq \mathcal{E}_{3}(F),
$$

by observing that the points of order 3 have one coordinate 0 . We prove the other inclusion. Since $\bigcup_{i=0}^{2} R_{i}$ is a group, it suffices to prove that the only constant points in the image of $\bigcup_{i=0}^{2} R_{i}$ under $\Psi^{-1}$ are points of order 3 . If $P=[X, Y, Z]$ is a point in

$$
\mathcal{E}(F) \cap \Psi^{-1}\left(\bigcup_{i=0}^{2} R_{i}\right),
$$

then $\Psi(P)$ is just the constant map, and we know it belongs to some $R_{i}$. If $i=1$ then the coordinate $Y$ must be 0 and so $P \in P_{1}$; if $i=2$ then $X=0$ and so $P \in P_{2}$; and if $i=0$ then $Z=0$ and so $P \in P_{0}$. Therefore $P$ is one of the nine points of order 3 . The first part of the lemma is proven.

We now prove the second part. From Lemma 5.1(i) we know that $\Psi\left(\left\{[n] \in \operatorname{End}_{F}(\mathcal{E}) \mid n \sim i\right\}\right) \subseteq R_{i}$. It is clear from Lemma 5.4 that $\Psi\left(U_{i}\right) \subseteq R_{i}$. This inclusion is actually an equality because the sets $U_{i}$ form a partition of $\operatorname{End}_{F}(\mathcal{E}) \oplus \mathcal{E}_{3}(F)$.

Consider the following equations:

$$
\begin{gathered}
x^{3}+y^{3}=1-t^{3}, \\
x^{3}+\left(1-t^{3}\right) y^{3}=1, \\
\left(1-t^{3}\right) x^{3}+y^{3}=1,
\end{gathered}
$$

and their analogues in projective coordinates

$\begin{array}{ll}(\mathrm{pMD}) & X^{3}+Y^{3}=\left(1-t^{3}\right) Z^{3}, \\ (\mathrm{pMD} 1) & X^{3}+\left(1-t^{3}\right) Y^{3}=Z^{3}, \\ (\mathrm{pMD} 2) & \left(1-t^{3}\right) X^{3}+Y^{3}=Z^{3} .\end{array}$

Each equation $\left(\mathrm{pMD} i\right.$ ) defines an elliptic curve $\mathcal{E}_{i}$ over $F(t)$. The point $[t, 1,1]$ is obviously a solution of (pMD1). Observe that each set $P_{i}$ is the set of constant points of the curve $\mathcal{E}_{i}$, and the points $P_{i}^{a}$ are of order 3 on $\mathcal{E}$, therefore also on the curves $\mathcal{E}_{i}$. Denote by $\mathcal{E}_{i}(F(t))$ the group of points of $\mathcal{E}_{i}$ which are rational over $F(t)$.

TheOREM 5.6. The disjoint union $\bigcup_{i=0}^{2} \mathcal{E}_{i}(F(t))$ of sets has a natural structure of a group, and

$$
\mathcal{E}_{i}(F(t))=\left\{\left[X_{n}, Y_{n}, Z_{n}\right] \oplus P_{j}^{a} \mid n+j \sim i \text { and } a=1, \xi \text { or } \xi^{2}\right\}
$$

for $i=0,1,2$. 
Proof. Consider the map

$$
\Phi: \bigcup_{i=0}^{2} \mathcal{E}_{i}(F(t)) \rightarrow \bigcup_{i=0}^{2} R_{i}
$$

defined by

$$
[X, Y, Z] \mapsto \begin{cases}f:[T, S, R] \rightarrow[X, Y, s Z] & \text { if }[X, Y, Z] \in \mathcal{E}_{0}(F(t)), \\ f:[T, S, R] \rightarrow[X, s Y, Z] & \text { if }[X, Y, Z] \in \mathcal{E}_{1}(F(t)), \\ f:[T, S, R] \rightarrow[s X, Y, Z] & \text { if }[X, Y, Z] \in \mathcal{E}_{2}(F(t)),\end{cases}
$$

where $t=T / R$ and $s=S / R$. This map is obviously a bijection of sets, and therefore $\Phi^{-1}$ brings the group structure from $\bigcup_{i=0}^{2} R_{i}$ onto $\bigcup_{i=0}^{2} \mathcal{E}_{i}(F(t))$. The second assertion of the theorem is an immediate consequence of Lemma 5.5: we have

$$
\mathcal{E}_{i}(F(t))=\Phi^{-1}\left(R_{i}\right)=\Phi^{-1} \circ \Psi\left(U_{i}\right)
$$

for $i=0,1,2$.

Consider the elliptic curve $\mathcal{E}^{0}$ defined by its affine equation

$$
y^{2}=4 x^{3}-1 \text {. }
$$

The curve $\mathcal{E}^{0}$ is isomorphic to $\mathcal{E}$ through the following isomorphism:

$$
\tau: \mathcal{E}^{0} \rightarrow \mathcal{E}, \quad[X, Y, Z] \mapsto[Y-\sqrt{3} Z,-Y-\sqrt{3} Z,-2 \sqrt{3} X] .
$$

Denote by $\mathcal{Q}$ the Weierstrass function on $\mathcal{E}^{0}$ (the reader who is not familiar with basic properties of the Weierstrass functions may consult [15]). Let $(\mathcal{P}, \mathcal{R})$ denote the affine part of $\tau\left(\left[\mathcal{Q}, \mathcal{Q}^{\prime}, 1\right]\right)$. We write briefly

$$
(\mathcal{P}, \mathcal{R})=\tau\left(\mathcal{Q}, \mathcal{Q}^{\prime}\right)
$$

If

$$
n=a+b \xi \in \operatorname{End}_{F}(\mathcal{E})
$$

we will write

$$
\bar{n}=a+b \xi^{2}
$$

for the conjugate of $n$. We have

$$
n \bar{n}=a^{2}+b^{2}-a b \in \mathbb{Z}
$$

and for $m, n \in \operatorname{End}_{F}(\mathcal{E})$, the obvious relations

$$
\overline{m+n}=\bar{m}+\bar{n} \quad \text { and } \quad \overline{m n}=\bar{m} \bar{n} .
$$

Denote by Id the identity map.

Lemma 5.7. We have

$$
\mathcal{P}^{\prime}=-\sqrt{3} \mathcal{R}^{2}
$$

and for any $[n] \in \operatorname{End}_{F}(\mathcal{E})$,

$$
[n](\mathcal{P}, \mathcal{R})=(\mathcal{P}, \mathcal{R}) \circ(\bar{n} \mathrm{Id}) .
$$


Proof. First we compute the derivative of $\mathcal{P}$. We get

$$
\mathcal{P}=\frac{\mathcal{Q}^{\prime}-\sqrt{3}}{-2 \sqrt{3} \mathcal{Q}} \quad \text { and } \quad \mathcal{R}=\frac{\mathcal{Q}^{\prime}+\sqrt{3}}{2 \sqrt{3} \mathcal{Q}}
$$

from $(\mathcal{P}, \mathcal{R})=\tau\left(\mathcal{Q}, \mathcal{Q}^{\prime}\right)$. Hence

$$
\mathcal{P}^{\prime}=\frac{-2 \sqrt{3} \mathcal{Q}^{\prime \prime} \mathcal{Q}+2 \sqrt{3}\left(\mathcal{Q}^{\prime}-\sqrt{3}\right) \mathcal{Q}^{\prime}}{12 \mathcal{Q}^{2}}=-\sqrt{3} \frac{\mathcal{Q}^{\prime \prime} \mathcal{Q}-\mathcal{Q}^{2}+\sqrt{3} \mathcal{Q}^{\prime}}{6 \mathcal{Q}^{2}} .
$$

From $\mathcal{Q}^{\prime 2}=4 \mathcal{Q}^{3}-1$ we get $2 \mathcal{Q}^{\prime \prime} \mathcal{Q}^{\prime}=12 \mathcal{Q}^{\prime} \mathcal{Q}^{2}$, hence $\mathcal{Q}^{\prime \prime}=6 \mathcal{Q}^{2}$. On the one hand, we replace $\mathcal{Q}^{\prime \prime}$ and $\mathcal{Q}^{\prime 2}$ in the expression of $\mathcal{P}^{\prime}$ to obtain

$$
\mathcal{P}^{\prime}=-\sqrt{3} \frac{6 \mathcal{Q}^{3}-\left(4 \mathcal{Q}^{3}-1\right)+\sqrt{3} \mathcal{Q}^{\prime}}{6 \mathcal{Q}^{2}}=-\sqrt{3} \frac{2 \mathcal{Q}^{3}+1+\sqrt{3} \mathcal{Q}^{\prime}}{6 \mathcal{Q}^{2}},
$$

and on the other hand we have

$$
-\sqrt{3} \mathcal{R}^{2}=-\sqrt{3}\left(\frac{\mathcal{Q}^{\prime}+\sqrt{3}}{2 \sqrt{3} \mathcal{Q}}\right)^{2}=-\sqrt{3} \frac{\mathcal{Q}^{\prime 2}+2 \sqrt{3} \mathcal{Q}^{\prime}+3}{12 \mathcal{Q}^{2}} ;
$$

hence

$$
-\sqrt{3} \mathcal{R}^{2}=-\sqrt{3} \frac{4 \mathcal{Q}^{3}-1+2 \sqrt{3} \mathcal{Q}^{\prime}+3}{12 \mathcal{Q}^{2}}=-\sqrt{3} \frac{2 \mathcal{Q}^{3}+\sqrt{3} \mathcal{Q}^{\prime}+1}{6 \mathcal{Q}^{2}},
$$

which proves the first assertion of the lemma.

Concerning the second assertion, it is known that for any $[n]^{0} \in \operatorname{End}_{F}\left(\mathcal{E}^{0}\right)$, we have

$$
[n]^{0}\left(\mathcal{Q}, \mathcal{Q}^{\prime}\right)=\left(\mathcal{Q}, \mathcal{Q}^{\prime}\right) \circ(n \mathrm{Id})
$$

(by construction of the Weierstrass function, see for example [15]). For $a=1$, $\xi$, or $\xi^{\prime}$, write $[a]^{0}$ for the automorphism on $\mathcal{E}^{0}$ defined by

$$
[a]^{0}[X, Y, Z]=[a X, Y, Z]
$$

(note the difference with the case of $\mathcal{E}$ ). It is easy to see that

$$
[\xi] \circ \tau=\tau \circ\left[\xi^{2}\right]^{0} .
$$

Therefore, if $n=p+q \xi$, we have

$$
\begin{aligned}
{[n] \circ \tau=[p+q \xi] \circ \tau } & :=([p] \oplus[q] \circ[\xi]) \circ \tau \\
& =[p] \circ \tau \oplus[q] \circ\left(\tau \circ\left[\xi^{2}\right]^{0}\right) \\
& =\tau \circ[p]^{0} \oplus \tau \circ[q]^{0} \circ\left[\xi^{2}\right]^{0}=\tau \circ[\bar{n}]^{0} .
\end{aligned}
$$

Combining the two equalities above, we find

$$
\begin{aligned}
{[n](\mathcal{P}, \mathcal{R}) } & =[n] \circ \tau\left(\mathcal{Q}, \mathcal{Q}^{\prime}\right)=\tau \circ[\bar{n}]^{0}\left(\mathcal{Q}, \mathcal{Q}^{\prime}\right) \\
& =\tau \circ\left(\mathcal{Q}, \mathcal{Q}^{\prime}\right) \circ(\bar{n} \mathrm{Id})=(\mathcal{P}, \mathcal{R}) \circ(\bar{n} \mathrm{Id}) .
\end{aligned}
$$

LEMMA 5.8. If $n \sim 1$, then

$$
\frac{x_{n}^{\prime}}{y_{n}^{2}}=\bar{n}
$$


Proof. Since $n \sim 1$, we know from Lemma 5.1(iv) and Lemma 5.7 that

$$
(\mathcal{P}, \mathcal{R}) \circ(\bar{n} \mathrm{Id})=[n](\mathcal{P}, \mathcal{R})=\left(x_{n} \circ \mathcal{P}, \mathcal{R} y_{n} \circ \mathcal{P}\right) .
$$

Therefore

$$
\begin{aligned}
x_{n}^{\prime} \circ \mathcal{P} & =\frac{1}{\mathcal{P}^{\prime}}\left(x_{n} \circ \mathcal{P}\right)^{\prime}=\frac{1}{-\sqrt{3} \mathcal{R}^{2}}(\mathcal{P} \circ \bar{n} \mathrm{Id})^{\prime} \\
& =\frac{1}{-\sqrt{3} \mathcal{R}^{2}} \bar{n} \mathcal{P}^{\prime} \circ \bar{n} \mathrm{Id}=\bar{n} \frac{\mathcal{R}^{2} \circ \bar{n} \mathrm{Id}}{\mathcal{R}^{2}} .
\end{aligned}
$$

Since $\mathcal{R}^{2} \circ \bar{n} \operatorname{Id}=\mathcal{R}^{2} y_{n}^{2} \circ \mathcal{P}$, we have $x_{n}^{\prime} \circ \mathcal{P}=\bar{n} y_{n}^{2} \circ \mathcal{P}$. The lemma follows because $(\mathcal{P}, \mathcal{R})$, seen as a map $\mathbb{C} \rightarrow \mathcal{E}$, is a global parametrization of the curve $\mathcal{E}$ (by construction of the Weierstrass function, see [15]).

Theorem 5.9. Let $n \in \mathbb{Z}[\xi]$. Then

$$
x_{n}^{3}(1)=\left\{\begin{array}{ll}
1 / \bar{n}^{3} & \text { if } n \sim 0, \\
1 & \text { if } n \sim 1, \\
-\bar{n}^{3} & \text { if } n \sim 2,
\end{array} \quad y_{n}^{3}(1)= \begin{cases}-1 / \bar{n}^{3} & \text { if } n \sim 0, \\
\bar{n}^{3} & \text { if } n \sim 1, \\
1 & \text { if } n \sim 2 .\end{cases}\right.
$$

Proof. Because of Lemma 5.2, it suffices to prove the statement for the $y_{n}$ 's. The proof is in two steps. Observe that if $x, y \in F(t)$ satisfy the equation $x^{3}+\left(1-t^{3}\right) y^{3}=1$, then $y$ cannot have a pole at 1 : suppose it had a pole of order $n$ at 1 ; then $\left(1-t^{3}\right) y^{3}$ would have a pole at 1 of order $3 n-1$, which would also be the order of $x^{3}$ at 1 , but this is impossible since $3 n-1$ is not a multiple of 3 .

Suppose that $n \sim 1$. From Lemma 5.1(iv), we know that

$$
x_{n}^{3}+\left(1-t^{3}\right) y_{n}^{3}=1 .
$$

This implies that $x_{n}^{3}(1)=1=y_{-n}^{3}(1)$. We get

$$
3 x_{n}^{\prime} x_{n}^{2}-3 t^{2} y_{n}^{3}+3\left(1-t^{3}\right) y_{n}^{\prime} y_{n}^{2}=0
$$

by differentiating both sides of (MD1). By Lemma 5.8 we know that $x_{n}^{\prime}=$ $\bar{n} y_{n}^{2}$. The equation becomes

$$
\bar{n} x_{n}^{2}-t^{2} y_{n}+\left(1-t^{3}\right) y_{n}^{\prime}=0
$$

after canceling the term $3 y_{n}^{2}$. Evaluating at $t=1$, we find

$$
y_{n}(1)=\bar{n} x_{n}^{2}(1)
$$

and therefore

$$
y_{n}^{3}(1)=\bar{n}^{3} x_{n}^{6}(1)=\bar{n}^{3} .
$$

Observe that for $n \sim 2$ we have $x_{n}(1)=-\bar{n} y_{n}^{2}(1)$.

Suppose now that $n \sim 0$ and write $n=m+a$, where $a$ is equal to 1 or $\xi$. Since $m \sim 2$ we get

$$
x_{m+a}(1)=\frac{y_{m}(1)}{a} \frac{1}{y_{m}^{2}(1)-a x_{m}(1)}
$$


from Lemma 5.1(v). Also we know from the previous step that

$$
x_{m}(1)=-\bar{m} y_{m}^{2}(1) \text {. }
$$

Therefore the equation above becomes

$$
\begin{aligned}
x_{m+a}(1) & =\frac{y_{m}(1)}{a} \frac{1}{y_{m}^{2}(1)+a \bar{m} y_{m}^{2}(1)}=\frac{1}{a y_{m}(1)} \frac{1}{1+a \bar{m}} \\
& =\frac{a}{y_{m}(1)} \frac{1}{a^{2}+\bar{m}}=\frac{a}{y_{m}(1)} \frac{1}{\overline{a+m}} .
\end{aligned}
$$

We know from the previous step that $y_{m}(1)^{3}=1$, and therefore

$$
x_{n}^{3}(1)=x_{m+a}^{3}(1)=\frac{1}{(\overline{a+m})^{3}}=\frac{1}{\bar{n}^{3}} .
$$

We obtain $y_{n}^{3}(1)$ from Lemma 5.2.

For $n+i \sim 1$, write (see the definition of $P_{i}^{a}$ before Lemma 5.4)

$$
\left(x_{n \oplus P_{i}^{a}}(t), s y_{n \oplus P_{i}^{a}}(t)\right)=[n](t, s) \oplus P_{i}^{a} .
$$

Corollary 5.10. For any $[n] \in \operatorname{End}_{F}(\mathcal{E})$ and $i=0,1,2$ such that $n+i \sim 1$ we have

$$
y_{n \oplus P_{i}^{a}}^{3}(1)=\bar{n}^{3} .
$$

Proof. From Lemma 5.4, we find

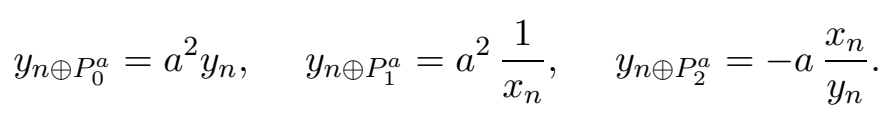

We conclude by applying Theorem 5.9.

Proof of Theorem 1.6. (a) Consider the following formula $\Psi_{0}(z)$, in the language $L_{3, \mathbb{Z}[t] \text {,Con,ord }}$

$$
\exists x, y\left(P_{3}(x) \wedge P_{3}(y) \wedge\left[1-(t+1)^{3}\right] y=1-x \wedge \operatorname{Con}(z) \wedge \operatorname{ord}(y-z)\right) .
$$

Apply Corollary 5.10 with $t$ replaced by $t+1$ to see that $\Psi_{0}(z)$ is equivalent to " $z \in \mathbb{Z}[\xi] \cap F$ and $z$ is a cube". The second difference of the three successive cubes $q-1, q$ and $q+1$ is

$$
\left[(q+1)^{3}-q^{3}\right]-\left[q^{3}-(q-1)^{3}\right]=6 q .
$$

Then the formula $\Psi_{1}(z)$, given by

$$
\exists z_{1}, z_{2}, z_{3}\left[\Psi_{0}\left(z_{1}\right) \wedge \Psi_{0}\left(z_{2}\right) \wedge \Psi_{0}\left(z_{3}\right) \wedge z=\left(z_{3}-z_{2}\right)-\left(z_{2}-z_{1}\right)\right]
$$

defines a set

$$
U=\left\{z \in F(t) \mid \Psi_{1}(z)\right\}
$$

which satisfies

$$
6 \mathbb{Z}[\xi] \cap F \subset U \subset \mathbb{Z}[\xi] \cap F .
$$


Finally, we get a positive-existential definition of $\mathbb{Z}[\xi] \cap F$ :

$$
z \in \mathbb{Z}[\xi] \cap F \Leftrightarrow \bigvee_{i=0}^{5} \exists w \Psi_{1}(w) \wedge z=w+i
$$

(b) Assume that for some $a, b \in \mathbb{Z}$ with $a b \neq 0, F$ has a subset $D$ such that for all $n \in \mathbb{Z}[\xi] \cap F$, there is a $d \in D$ such that

$$
a n^{3}+b d^{3}=1 .
$$

Replace each occurrence of $\operatorname{Con}(z)$ in the proof of (a) by the formula

$$
\theta(z): \quad \exists w\left[P_{3}(z) \wedge P_{3}(w) \wedge a z+b w=1\right] .
$$

Then the proof of (a) still works. This is, first, because the curve

$$
a X^{3}+b Y^{3}=1
$$

is of genus 1 and does not admit a rational parametrization (by Hurwitz's formula, see [3]), hence any $z$ satisfying $\theta(z)$ must be in $F$, and secondly because, by assumption, for any $n \in \mathbb{Z}[\xi] \cap F, \theta\left(n^{3}\right)$ holds.

\section{References}

[1] M. Davis, Hilbert's tenth problem is unsolvable, Amer. Math. Monthly 80 (1973), 233-269.

[2] J. Denef, The Diophantine Problem for polynomial rings and fields of rational functions, Trans. Amer. Math. Soc. 242 (1978), 391-399.

[3] R. Hartshorne, Algebraic Geometry, Grad. Texts in Math. 52, Springer, 1977.

[4] K. H. Kim and F. W. Roush, Diophantine undecidability of $\mathbb{C}\left(t_{1}, t_{2}\right)$, J. Algebra 150 (1992), 35-44.

[5] S. Lang, Elliptic Functions, Grad. Texts in Math. 112, Springer, New York, 1987.

[6] —, Hyperbolic diophantine analysis, Bull. Amer. Math. Soc. 14 (1986), 159-205.

[7] L. Lipshitz, Quadratic forms, the five square problem, and diophantine equations, in: The Collected Works of J. Richard Büchi (S. MacLane and D. Siefkes, eds.), Springer, 1990, 677-680.

[8] Yu. Matiyasevich, Enumerable sets are diophantine, Dokl. Akad. Nauk SSSR 191 (1970), 279-282 (in Russian); English transl.: Soviet Math. Dokl. 11 (1970), 354-358.

[9] B. Mazur, Questions of decidability and undecidability in number theory, J. Symbolic Logic 59 (1994), 353-371.

[10] T. Pheidas, Hilbert's Tenth Problem for fields of rational functions over finite fields, Invent. Math. 103 (1991), 1-8.

[11] - Undecidability of existential theories of rings and fields: A survey, in: Contemp. Math. 270, Amer. Math. Soc., 2000, 49-106.

[12] E. S. Selmer, The Diophantine equation $a x^{3}+b y^{3}+c z^{3}=0$, Acta Math. 85 (1951), 203-362; 92 (1954), 191-197.

[13] A. Shlapentokh, Diophantine undecidability over algebraic function fields over finite fields of constants, J. Number Theory 58 (1996), 317-342.

[14] - Hilbert's tenth problem over number fields, a survey, in: Contemp. Math. 270, Amer. Math. Soc., 2000, 107-137. 
[15] J. H. Silverman, The Arithmetic of Elliptic Curves, Grad. Texts in Math. 106, Springer, 1986.

[16] C. R. Videla, Hilbert's Tenth Problem for rational function fields in characteristic 2, Proc. Amer. Math. Soc. 120 (1994), 249-253.

[17] P. Vojta, Diagonal quadratic forms and Hilbert's Tenth Problem, in: Contemp. Math. 270, Amer. Math. Soc., 2000, 261-274.

[18] —, Diophantine Approximations and Value Distribution Theory, Lecture Notes in Math. 1239, Springer, 1987.

[19] K. Zahidi, The existential theory of real hyperelliptic function fields, J. Algebra 233 (2000), 65-86.

[20] - Hilbert's Tenth Problem for rings of rational functions, Notre Dame J. Formal Logic 43 (2002), 181-192.

Department of Mathematics

University of Crete

71409 Heraklion, Crete, Greece

E-mail: pheidas@math.uoc.gr
Departamento de Matemática Facultad de Ciencias Físicas y Matemáticas

Universidad de Concepción Casilla 160C Concepción, Chile E-mail: xvidaux@udec.cl

Received 27 September 2004;

in revised form 17 March 2005 